\title{
Isolation and characterization of anti-tumor compounds from ethyl acetate extract of Rumex japonicus houtt roots and their cytotoxic effects
}

\author{
Zhaogen $\mathrm{WU}^{1 \ddagger}$, Kashif AMEER ${ }^{2 \ddagger}$, Guihun JIANG ${ }^{1 *}$ (it)
}

\begin{abstract}
To isolate and characterize anti-tumor compounds through nuclear magnetic resonance (NMR) spectroscopy of ethyl acetate extract of R. japonicus Houtt root and their cytotoxic effects were evaluated in terms of measuring inhibition of growth of three cancer cell lines: human hepatoma cells (HepG2), cerebral glioma cells (U251) and ovarian carcinoma cells (SK-OV-3/DDP). The roots of $R$. japonicus Houtt were extracted with ethanol, and partitioned with petroleum ether, dichloromethane and ethyl acetate, respectively. The ethyl acetate extract was separated chromatographically using macroporous resin, positive and negative phase silica gel and high performance liquid chromatography. The structures of isolated compounds were elucidated by nuclear magnetic resonance (NMR) spectral data. Ten compounds were isolated form the ethyl acetate extract of $R$. japonicus roots. Their structures were determined as emodin (1), chrysophanol (2), aloe emodin (3), physcion (4), quercetin (5), resveratrol (6), $\beta$-sitosterol (7), $3 \beta$-acetoxy-28-hydroxyurs-12-ene (8), sucrose (9) and dibutyl phthalate (10). Among of them, compounds 1 , $3,4,6$ and 8 exhibited highly significant $(p<0.05)$ cytotoxicities against HepG2 to a certain extent. Compounds 3-6 exhibited moderate cytotoxicities against $\mathrm{U} 251$ with $\mathrm{IC}_{50}$ values of $19.8,23.5,23.8$ and $17.8 \mu \mathrm{M}$, respectively. Compounds 1,2 and 7 also exhibited cytotoxicities against $\mathrm{U} 251$ to a certain extent with $\mathrm{IC}_{50}$ values of $48.3,50.1$ and $85.3 \mu \mathrm{M}$, respectively. Compounds 1-6 exhibited moderate cytotoxicities against (SK-OV-3/DDP) with $\mathrm{IC}_{50}$ values of 62.8, 64.2, 31.3, 61.0, 47.4 and $42.0 \mu \mathrm{M}$, respectively.
\end{abstract}

Keywords: Rumex japonicus Houtt; ethyl acetate extract; chemical constituents; anti-tumor activity; NMR; characterization.

Practical Application: Ethyl acetate extract of R. japonicus Houtt root Isoltaion, NMR charcatrization and cytotoxic effects were evaluated on three cancer cell lines (HepG2, U251, SK-OV-3/DDP).

\section{Introduction}

Since prehistoric times, human beings have been using plants as folkloric sources of phytoconstituents of medicinal and pharmaceutical significance. In modern medicine age, single-compound based purified drugs have been emerged with promising potential as anti-tumor agents (Jenks \& Smigel, 1995). Plant-derived chemical compounds and their semisynthetic and synthetic analogues are in use by pharmaceutical and oncology researchers. Since, 1961, United Stated Food and Drug Administration (FDA) has approved plant-derived anti-cancer and anti-tumor chemicals to prepare anti-cancer drugs (Lee, 1999). As perennial herb, Rumex japonicus Houtt belongs to the Polygonaceae family and is known in Traditional Chinese Medicine (TCM) as Yang-Ti. It is widely distributed in Eastern China, Central China, Southern China, Korea and Japan (Xie \& Yang, 2014). Rumex japonicus Houtt root has medicinal significance and in folklore and alternate medicines, it is employed for various medicinal benefits like as a antitumor, anti-inflammatory, purgative and detox agent and as an alternate medicine for skin treatment, scabies, regulation of blood circulation and wastes clearance out of body (Lee et al., 2006; Xie \& Yang, 2014). Moreover, $R$. japonicus Houtt comprises of rich quantities of several phytoconstituents of pharmaceutical significance, such as oxanthrones, anthraquinones, nepodin, rutin and phenolic compounds in its roots (Zhou et al., 2005).

Cancer is one of the main chronic diseases that affect the health of people worldwide including China. Globally, cancer is categorized as the second leading death cause next to cardiovascular diseases. Lung cancer is one of the primary cancer types as cause of death in developing countries and nearly $85 \%$ cases of lung cancer are reported because of smoking as the main factor. As far as the mortality rate of females and males is concerned, colon cancer is the second most widely known death cause. (Thakurta et al., 2015). Colon cancer affects almost one million people every year worldwide. On the other hand, each year about 10,000 cases of malignant gliomas are reported in United States only (Alwarsamy \& Ravichandran, 2011).

The treatment and prevention of cancer has always been a difficult problem for researchers all over the world (Lichota \& Gwozdzinski, 2018). If not effectively controlled, metastatic cancer cell will cause tissue failure and necrosis, thus threatening life (Steenkamp \& Gouws, 2006). Adjunct therapies including cisplatin, interferon alpha epirubicin and doxorubicin are commonly used to overcome abnormal growth of cancer 
cells, however, these chemotherapy agents are ineffective against resistance mechanism of cancer cells owing to up-regulation of multi-drug resistant proteins (MDR) and lower levels of apoptotic proteins. Therefore, more effective antitumor and tumor suppressions chemotherapy agents are needed to effectively overcome apoptotic induction for successfully treating cancer (Machana et al., 2012).

At present, there are many studies available on inhibitory and therapeutic effects of TCM on cancer cells. Therefore, pure plant extracts are of great significance for cancer treatment as natural alternative of synthetic and semi-synthetic chemotherapy agents. Until now, there have been few systematic studies on in-vitro anti-tumor effects of $R$. japonicus Houtt root extracts (He et al., 2016; Xie \& Yang, 2014; Yang et al., 2019).

Therefore, the objective of this study was to isolate and characterize anti-tumor compounds through nuclear magnetic resonance (NMR) spectroscopy of ethyl acetate extract of R. japonicus Houtt root and their cytotoxic effects were evaluated in terms of measuring inhibition of growth of three cancer cell lines (HepG2, U251, SK-OV-3/DDP).

\section{Materials and methods}

\subsection{Materials}

Roots of Rumex japonicus Houtt were collected in nearby mountain area of Jilin City, Jilin province, China. After collection, the roots of Rumex japonicus Houtt were subjected to drying in conjunction with active ventilation at normal room temperature of $25^{\circ} \mathrm{C}$. Then, polyethylene bags were utilized to pack the dried $R$. japonicus roots followed by $-80^{\circ} \mathrm{C}$ prior to further use.

\subsection{Extraction and isolation of Rumex japonicus compounds}

Dried roots were amounted to $10 \mathrm{~kg}$ after drying. Dried R. japonicus Houtt roots were subjected to ethanol (95\%) extraction separately extracted and total extraction time was $24 \mathrm{~h}$. After extraction time completion, the root extracts were filtered using Whatman' filter paper No. 2 (Whatman Int. Ltd., Maidstone, England), and concentrated in vacuum at $40{ }^{\circ} \mathrm{C}$. Concentrates $(546.8 \mathrm{~g}$ ) were then suspended with distilled water and petroleum ether (4 L, 3 times) $(78.5 \mathrm{~g}$ ) was employed for partition in successive manner: dichloromethane ( $4 \mathrm{~L}, 3$ times) (138.4 g), and ethyl acetate (4 L, 3 times) (185.6 g); Flow chart of Rumex japonicus Houtt roots extraction is depicted in in Figure 1. Ethyl acetate extract $(150 \mathrm{~g})$ from the roots of $R$. japonicus Houtt was eluted with macroporous resin. Eluents were water, $20 \%$ ethanol, $40 \%$ ethanol and $95 \%$ ethanol, respectively. The collected components were concentrated to obtain water fraction $(13.7 \mathrm{~g})$, $20 \%$ ethanol fraction $(55.6 \mathrm{~g})(\mathrm{YT} 1), 40 \%$ ethanol fraction $(48.0 \mathrm{~g})$ (YT2) and 95\% ethanol fraction (25.7 g) (YT3).

Column chromatography aided with Merck silica gel (mesh size ranges $60,200-300,10$ i. d. $\times 40 \mathrm{~cm}$ ) was employed as means for YT1 fraction (55.6 g) elution with a ethyl acetate/ methanol (100:0-0:100) to give 4 fractions (YT1-1-YT1-4). Same Merck silica gel was utilized for column chromatographic separation of YT1-1 fraction ( $7.8 \mathrm{~g}$ ) by eluting with a petroleum ether/ethyl acetate (100:1-1:1) to give 7 fractions (YT1-1-1YT1-1-7). Compound 9 (3.4 mg) was obtained from YT1-1-2 petroleum ether/ethyl acetate (100:1-1:1) fraction (1.8 g). YT1-1-5 fraction $(85.6 \mathrm{mg}$ ) was processed to get YT1-1-5-2 fraction $(25 \mathrm{mg})$. Compound 3 (12.5 $\mathrm{mg}$ ) was obtained from YT1-1-5-2 fraction using medium-pressure preparative liquid chromatograph eluting with methanol/water (1:1-3:1).

Moreover, 10 fractions (YT1-3-1-YT1-3-10) of YT1-3 fraction $(19.8 \mathrm{~g})$ were obtained through silica gel-aided column chromatography as per TLC results. Among them, the YT1-3-1 fraction was needle crystal, and after recrystallization repeatedly to give compound 1 (9.5 mg). Similarly, YT1-3-5-6 fraction (220.5 mg) was obtained from YT1-3-5 fraction $(3.5 \mathrm{~g}$ ) separation through silica gel-based column chromatography. Then, compound $4(6.9 \mathrm{mg})$ recovery was carried out using medium-pressure preparative liquid chromatograph by elution through methanol/ water (4:1-8:1). YT1-3-8 fraction (2.2 g) was used to give YT1-38-5 fraction ( $95 \mathrm{mg})$. Then, compound $2(15.3 \mathrm{mg}$ ) was obtained through MPPLC elution with methanol/water (4:1-8:1).

YT1-4 fraction (8.6 g) was used to give 6 fractions (YT1-41-YT1-4-6) petroleum ether/ethyl acetate (50:1-1:1) followed by recovery of compound 6 from YT1-4-5 (178.5 mg) group by MPPLC with methanol/water (1:1-3:1) obtained compound 6 (8.2 mg). YT2 fraction (48 g) yielded 4 fractions (YT2-1-YT2-4), YT2-2 fraction (18.4 g) yielded 8 fractions (YT2-2-1-YT2-2-8), YT2-2-3 fraction (3.6 g) yielded YT2-2-3-1 fraction (280.5 mg), YT2-3 fraction ( $15 \mathrm{~g}$ ) yielded 8 fractions (YT2-3-1-YT2-3-10), YT2-3-2 fraction $(654.5 \mathrm{mg})$ yielded YT2-3-2-3 fraction (25.8 mg), YT2-3-5 fraction (1.2 g) yielded YT2-3-5-2 fraction ( $34.5 \mathrm{mg})$. Compounds 7 and 10 were obtained from these fractions through gradiet elution. The obtain fraction was eluted with a MeOH-H2O (4:1-8:1) to give YT2-2-31-3 fraction $(85.6 \mathrm{mg})$ based on TLC results. The fraction continue eluted with a $\mathrm{MeOH}-\mathrm{H} 2 \mathrm{O}$ (2:1) to obtain compound 5 (6.4 mg). Similarly, YT3 fractions (YT3-1-YT3-3) and YT3-2 fractions (YT32-1-YT3-2-5) according to TLC results were obtained. The YT3-2-3 fraction $(982.5 \mathrm{mg}$ ) eluting with a petroleum ether / ethyl acetate (50:1-1:1) to give YT3-2-3-1 fraction (53.6 $\mathrm{mg}$ ), and the continue eluted with a MeOH-H2O (1:1-4:1) to give compound 8 (2.6 mg).

\subsection{NMR measurement for structural analysis}

Different NMR experimentation modalities were carried out, such as ${ }^{1} \mathrm{H}$ NMR, APT, J-resolved, ${ }^{1} \mathrm{H}-{ }^{1} \mathrm{H}$ COSY, TOCSY, ${ }^{1} \mathrm{H}-{ }^{13} \mathrm{C}$ HMBC, ${ }^{1} \mathrm{H}-{ }^{13} \mathrm{C}$ HSQC, and NOESY at $600 \mathrm{MHz}$ using a Micromass mass spectrometer (Synapt, Waters, USA). TMS was utilized as a standard (Maltese et al., 2009).

\subsection{Antitumor activity of cancer cells (HepG2, U251, $S K-O V-3 / D D P$ )}

Antitumor activity of HepG2, U251, SK-OV-3/DDP cancer cells were determined by reported methods of Qin et al. (2017, 2019) and Emerson et al. (2007). Tumor cell lines of human beings, known as HepG2, U251 and SK-OV-3/DDP, along with normal liver cell line HL-7702 were purchased from the Shanghai Cell Bank of Chinese Academy of Sciences. Cells cultures were prepared in Dulbecco's modified Eagle's medium (DMEM) followed by $10 \%$ supplementation of fetal bovine serum (FBS) 


\section{Rumex Japonicus Houtt roots (10 kg)}

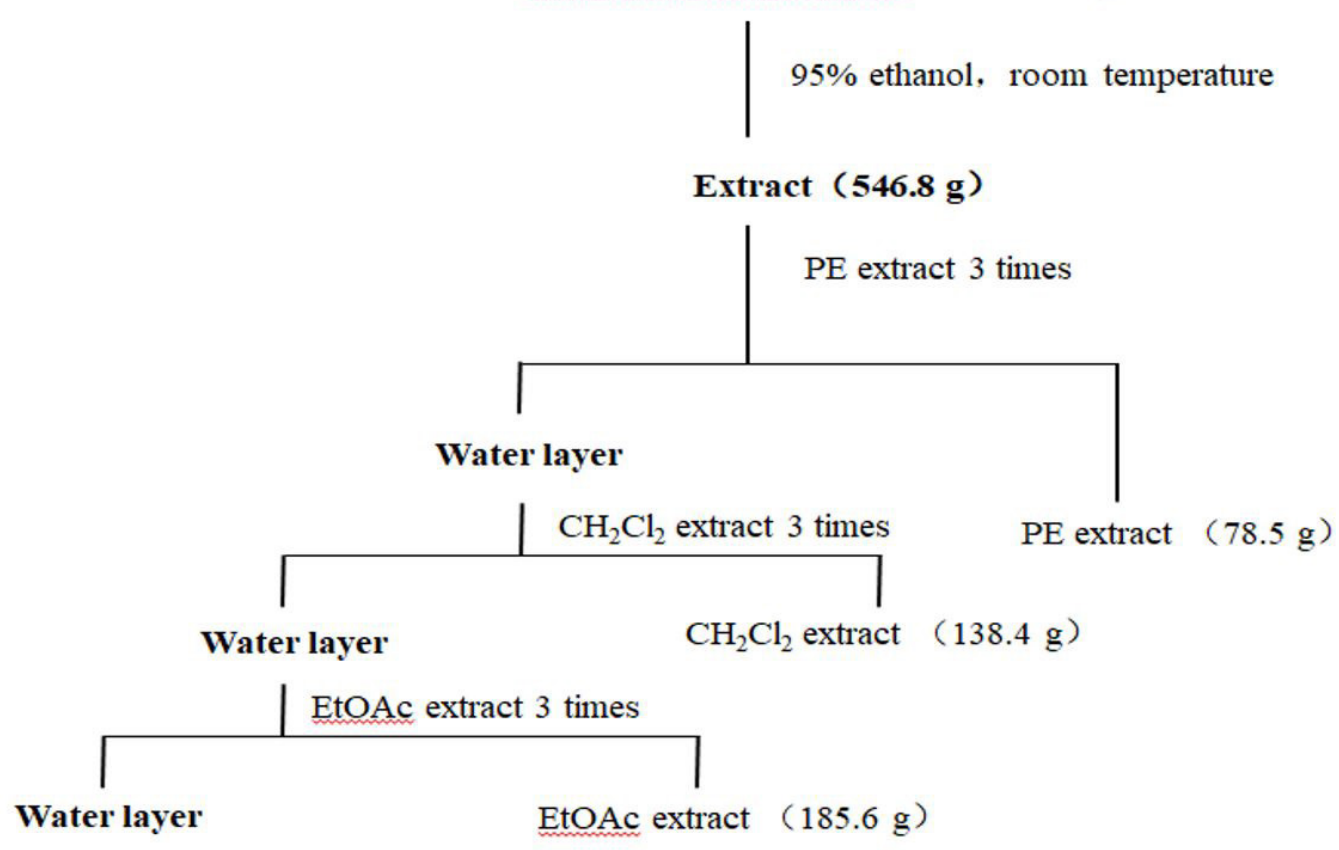

Figure 1. Flow chart of Rumex japonicus Houtt roots extraction.

along with $100 \mathrm{U} / \mathrm{mL}$ penicillin and streptomycin. Cell cultures were subjected to atmospheric humidification at $37^{\circ} \mathrm{C}$ with combination of $95 \%$ air and $5 \% \mathrm{CO}_{2}$. Evaluating extracts and compounds $(2.0 \mathrm{mM})$ were prepared through DMSO stock solution method prior to dilution to make working concentrations of $0.5 \%$ DMSO-containing PBS buffer. Whereas, cisplatin was employed as positive control and $0.9 \%$ sodium chloride was utilized for dissolution.

\subsection{Statistical analysis}

Duplicate experiments were carried out for all measurements and values were recorded in triplicate manner. Means \pm standard deviations were calculated for all measurements. SPSS software (SPSS Inc., Chicago, IL, USA) version 18.0 was the software package utilized for data analysis consisting of one-way analysis of variance (ANOVA). Duncan's multiple-range test was utilized to evaluate significance at $p<0.05$.

\section{Results and discussion}

\subsection{Yields and structure of isolated compounds}

Yields of compounds isolated from roots of Rumex japonicus Houtt (mg/g on dry weight basis of fractions) were determined. Table 1 shows that chrysophanol was the highest compound ( 20\%) present in EtOH fraction. Aloe emodin, emodin, physcion, quercetin, resveratrol, dibutyl phthalate were found relatively in significant quantities ranging from 20 to $40 \%$ in $\mathrm{EtOH}$ fractions. The yields of isolated chemical compounds fractions from $R$. japonicus Houtt root, such as aloe emodin, chrysophanol, emodin, physcion, quercetin, resveratrol, $\beta$-sitosterol, $3 \beta$-acetoxy-
Table 1. Yields of compounds isolated from roots of Rumex japonicus Houtt (mg/g dry wt of fractions).

\begin{tabular}{lc}
\hline Compounds & Yield (mg/g dry wt. of fractions) \\
\hline Aloe emodin & 9.5 \\
Chrysophanol & 15.3 \\
Emodin & 12.5 \\
Physcion & 6.9 \\
Quercetin & 6.4 \\
Resveratrol & 8.2 \\
$\beta$-sitosterol & 2.8 \\
$3 \beta$-Acetoxy-28-hydroxyurs-12-ene & 2.6 \\
Sucrose & 3.4 \\
Dibutyl phthalate & 9.8 \\
\hline
\end{tabular}

28-hydroxyurs-12-ene, sucrose and dibutyl phthalate were 9.5, $15.3,12.5,6.9,6.4,8.2,2.8,2.6,3.4$, and $9.8 \mathrm{mg} / \mathrm{g}$, respectively on dry weight (DW) basis (Table 1). Similar findings have been reported by Guo et al. (2008) whereby a liverwort plant (Scapania verrucosa) was screened for antitumor compounds. The etherextract based yield of dried S. verrucosa was $0.6 \%$ and about 59 compounds were isolated from S. verrucosa. Flow chart for isolation of ethyl acetate extract from Rumex japonicus Houtt roots is shown in Figure 2.

The structures of compounds showed in Figure 3 elucidated by ${ }^{1} \mathrm{H}-\mathrm{NMR}$ and ${ }^{13} \mathrm{C}-\mathrm{NMR}$ analyses are given below as follows:

Compound 1: Aloe emodin, yellow needle crystal, ${ }^{1} \mathrm{H}-\mathrm{NMR}$ (500 MHz,DMSO): $\delta_{\mathrm{H}}(\mathrm{ppm}) 7.86(1 \mathrm{H}, \mathrm{t}, J=7.9 \mathrm{~Hz}, \mathrm{H}-6), 7.77$ $(1 \mathrm{H}, \mathrm{d}, J=7.5 \mathrm{~Hz}, \mathrm{H}-7), 7.74(1 \mathrm{H}, \mathrm{s}, \mathrm{H}-4), 7.44(1 \mathrm{H}, \mathrm{d}, J=9.5 \mathrm{~Hz}, \mathrm{H}-$ 5), $7.35(1 \mathrm{H}, \mathrm{s}, \mathrm{H}-2), 12.00(1 \mathrm{H}, \mathrm{s}, \mathrm{OH}), 4.69(2 \mathrm{H}, \mathrm{d}, J=6.0 \mathrm{~Hz}, \mathrm{H}-$ 1'). ${ }^{13} \mathrm{C}-\mathrm{NMR}$ (125 MHz,DMSO): $\delta \mathrm{C}$ (ppm) 192.1 (C-9),181.9 
EtOAc extract roots of the Rumex Japonicus Houtt

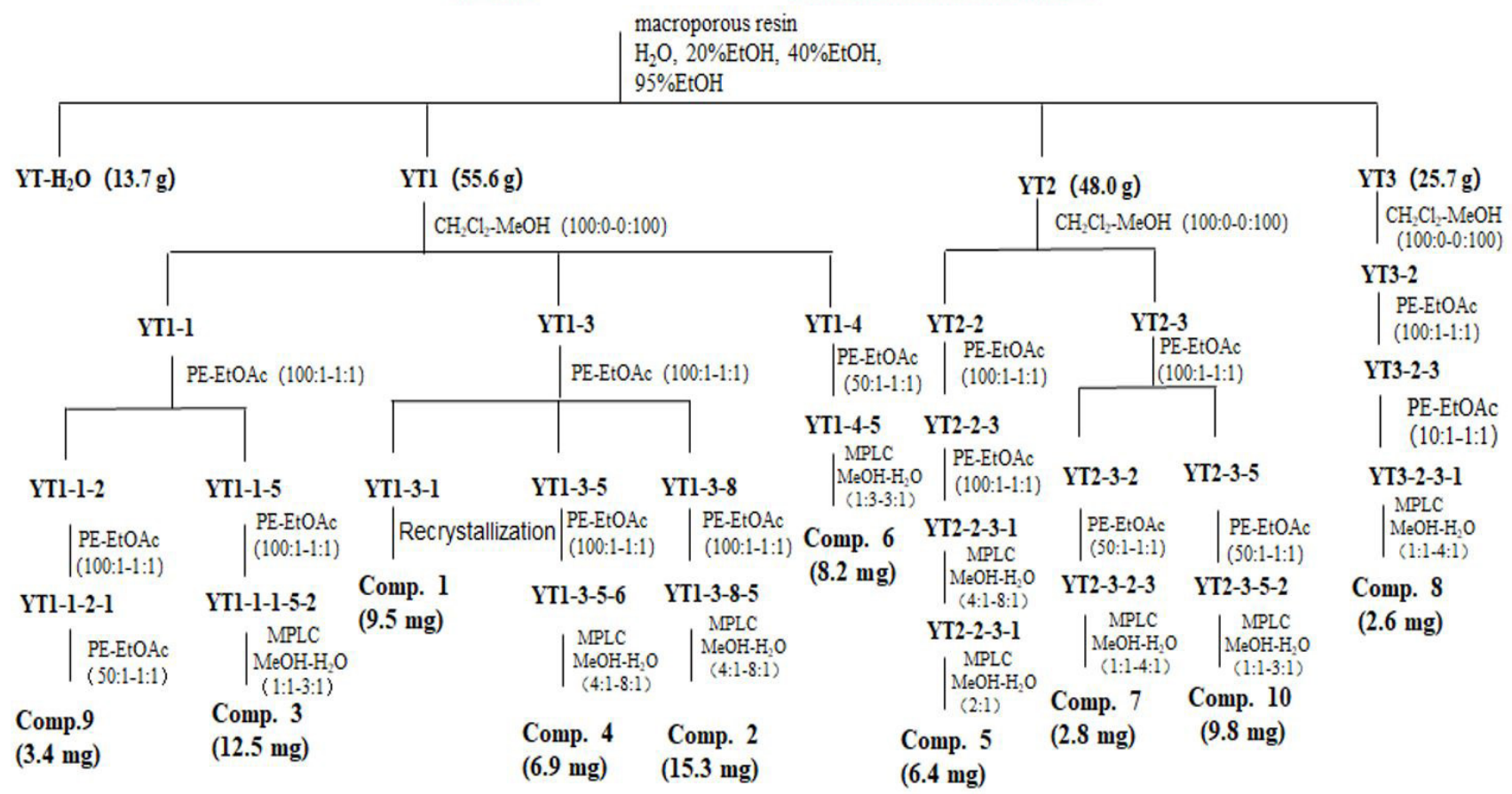

Figure 2. Flow chart for isolation of ethyl acetate extract from Rumex japonicus Houtt roots.

(C-10), 162.1 (C-8), 161.8 (C-1),154.2 (C-3),133.8 (C-5a), 133.6 (C-4a),124.9 (C-5),122.9 (C-4),121.1 (C-6), 119.8 (C-2), 117.6 (C-7), 116.4 (C-1a), 114.9 (C-8a), 62.5 (C-1').

Compound 2: Chrysophanol, yellow needle crystal, ${ }^{1} \mathrm{H}-\mathrm{NMR}(500 \mathrm{MHz}, \mathrm{DMSO}): \delta_{\mathrm{H}}(\mathrm{ppm}) 11.95$ $(1 \mathrm{H}, \mathrm{s}, 1-\mathrm{OH}), 7.81(1 \mathrm{H}, \mathrm{d}, J=7.0 \mathrm{~Hz}, \mathrm{H}-5), 7.72(1 \mathrm{H}, \mathrm{d}, J=$ $7.5 \mathrm{~Hz}, \mathrm{H}-6), 7.57(1 \mathrm{H}, \mathrm{d}, J=1.5 \mathrm{~Hz}, \mathrm{H}-4), 7.39(1 \mathrm{H}, \mathrm{d}, J=8.3 \mathrm{~Hz}, \mathrm{H}-$ 7), $7.23(1 \mathrm{H}, \mathrm{d}, J=1.5 \mathrm{~Hz}, \mathrm{H}-2), 2.45\left(3 \mathrm{H}, \mathrm{s},-\mathrm{CH}_{3}\right) .{ }^{13} \mathrm{C}-\mathrm{NMR}$ (125 MHz,DMSO): $\delta \mathrm{C}$ (ppm) 192.1(C-9),181.9 (C-10),162.0 (C-1),161.8 (C-8), 149.6 (C-3), 137.8 (C-6), 134.1 (C-5a), 133.8 (C-4a),124.9 (C-2),124.6 (C-7),121.0 (C-4),119.8 (C-5), 116.3 (C-1a), 112.9 (C-8a), $21.2\left(3-\mathrm{CH}_{3}\right)$.

Compound 3: Emodin, aurantium needle crystal, ${ }^{1} \mathrm{H}-\mathrm{NMR}$ (500 MHz,DMSO): $\delta_{\mathrm{H}}$ (ppm) $12.04(1 \mathrm{H}, \mathrm{s}, 1-\mathrm{OH}), 11.96(1 \mathrm{H}, \mathrm{s}, 8-$ $\mathrm{OH}), 7.42(1 \mathrm{H}, \mathrm{d}, J=2.2 \mathrm{~Hz}, \mathrm{H}-4), 7.11(1 \mathrm{H}, \mathrm{d}, J=2.1 \mathrm{~Hz}, \mathrm{H}-5), 7.07$ $(1 \mathrm{H}, \mathrm{d}, J=2.2 \mathrm{~Hz}, \mathrm{H}-2), 6.56(1 \mathrm{H}, \mathrm{d}, J=2.1 \mathrm{~Hz}, \mathrm{H}-7), 2.39$ (3H,s,3$\left.\mathrm{CH}_{3}\right) \cdot{ }^{13} \mathrm{C}-\mathrm{NMR}(125 \mathrm{MHz}, \mathrm{DMSO}): \delta \mathrm{C}$ (ppm) 190.1 (C-9), 181.8 (C-10),166.0 (C-6),164.9 (C-8), 161.9 (C-1),148.7 (C-3),135.5 (C-5a),133.2 (C-4a), 124.6 (C-2), 120.9 (C-4),113.8 (C-1a) 109.4 (C-5), 109.2 (C-8a), $108.4(\mathrm{C}-7), 21.2\left(3-\mathrm{CH}_{3}\right)$.

Compound 4: Physcion, aurantium needle crystal, ${ }^{1} \mathrm{H}-\mathrm{NMR}$ (500 MHz,DMSO): $\delta_{\mathrm{H}}(\mathrm{ppm}) 12.13(1 \mathrm{H}, \mathrm{s}, 8-\mathrm{OH}), 7.55$ (1H,d,J $=2.3 \mathrm{~Hz}, \mathrm{H}-4), 7.22(1 \mathrm{H}, \mathrm{d}, J=2.3 \mathrm{~Hz}, \mathrm{H}-2), 7.21(1 \mathrm{H}, \mathrm{d}, J=$ $2.5 \mathrm{~Hz}, \mathrm{H}-5), 6.90(1 \mathrm{H}, \mathrm{d}, J=2.5 \mathrm{~Hz}, \mathrm{H}-7), 3.94\left(3 \mathrm{H}, \mathrm{s}, 6-\mathrm{OCH}_{3}\right)$, $2.44\left(3 \mathrm{H}, \mathrm{s}, 3-\mathrm{CH}_{3}\right) .{ }^{13} \mathrm{C}-\mathrm{NMR}(125 \mathrm{MHz}, \mathrm{DMSO}): \delta \mathrm{C}(\mathrm{ppm})$ 190.5 (C-9), 181.8 (C-10), 166.7 (C-8), 164.9 (C-1), 161.9 (C6),149.1 (C-3), 135.4 (C-5a),133.4 (C-4a),124.7 (C-2), 121.1 (C-4),114.0 (C-1a),108.1 (C-5),108.1 (C-8a),107.1 (C-7), 56.9 $\left(6-\mathrm{OCH}_{3}\right), 22.0\left(3-\mathrm{CH}_{3}\right)$.
Compound 5: Quercetin, yellow needle crystal, ${ }^{1} \mathrm{H}-\mathrm{NMR}$ (500MHz,DMSO): $\delta_{\mathrm{H}}(\mathrm{ppm}) 12.46(5-\mathrm{OH}), 7.66(1 \mathrm{H}, \mathrm{d}, J$ $=2.1 \mathrm{~Hz}, \mathrm{H}-2$ '),7.53 (1H,dd $J=8.5 \mathrm{~Hz}, J=2.1 \mathrm{~Hz}, \mathrm{H}-6$ '),6.89 $\left(1 \mathrm{H}, \mathrm{d}, J=8.5 \mathrm{~Hz}, \mathrm{H}-5^{\prime}\right), 6.41(1 \mathrm{H}, \mathrm{d}, J=2.0 \mathrm{~Hz}, \mathrm{H}-8), 6.19(1 \mathrm{H}, \mathrm{d}, J$ $=2.0 \mathrm{~Hz}, \mathrm{H}-6) .{ }^{13} \mathrm{C}-\mathrm{NMR}(125 \mathrm{MHz}, \mathrm{DMSO}): \delta \mathrm{C}$ (ppm) 176.3 (C-4), 164.3 (C-7),161.2 (C-5),156.7 (C-9),148.2 (C-4'),145.5 (C-3'),122.5 (C-1'), 120.5 (C-6'),116.1 (C-5'), 115.5 (C-2'), 103.5 (C-10),98.7 (C-6),93.9 (C-8),147.3 (C-2),136.2 (C-3).

Compound 6: Resveratrol, white needle crystal, ${ }^{1} \mathrm{H}-\mathrm{NMR}$ (500MHz,CD 3 OD): $\delta_{\mathrm{H}}(\mathrm{ppm}) 7.04(1 \mathrm{H}, \mathrm{d}, J=16.3 \mathrm{~Hz}, \mathrm{H}-7), 6.90$ $(1 \mathrm{H}, \mathrm{d}, J=16.3 \mathrm{~Hz}, \mathrm{H}-8), \delta_{\mathrm{H}}(\mathrm{ppm}) 7.43(2 \mathrm{H}, \mathrm{d}, J=8.4 \mathrm{~Hz}, \mathrm{H}-2,6), 6.84$ $(2 \mathrm{H}, \mathrm{d}, J=8.4 \mathrm{~Hz}, \mathrm{H}-3,5), 6.53(2 \mathrm{H}, \mathrm{d}, J=2.2 \mathrm{~Hz}, \mathrm{H}-10,14), 6.24(1 \mathrm{H}, \mathrm{t}, J$ $=2.2 \mathrm{~Hz}, \mathrm{H}-12) .{ }^{13} \mathrm{C}-\mathrm{NMR}\left(125 \mathrm{MHz}, \mathrm{CD}_{3} \mathrm{OD}\right): \delta \mathrm{C}(\mathrm{ppm}) 158.3(\mathrm{C}-$ 11,13),156.9 (C-1),139.9 (C-4),129.0 (C-9),127.4 (C-3,5),115.1 (C2,6),104.4 (C-10,14),101.3 (C-12), \&C (ppm) 128.0 (C-7), 125.6(C-8).

Compound 7: $\beta$-sitosterol, white needle crystal, ${ }^{1} \mathrm{H}-\mathrm{NMR}(300$ $\left.\mathrm{MHz}, \mathrm{CDCl}_{3}\right): \delta_{\mathrm{H}}(\mathrm{ppm}) 5.34(1 \mathrm{H}, \mathrm{d}, J=5.0 \mathrm{~Hz}, \mathrm{H}-6), 3.49(\mathrm{~m}, \mathrm{H}-3)$, 0.67 (3H,s,H-18), 1.01 (3H,s,H-19), $0.84(3 \mathrm{H}, \mathrm{d}, J=6.0 \mathrm{~Hz}, \mathrm{H}-21)$, $0.92(3 \mathrm{H}, \mathrm{d}, J=7.6 \mathrm{~Hz}, \mathrm{H}-26), 0.91(3 \mathrm{H}, \mathrm{d}, J=7.6 \mathrm{~Hz}, \mathrm{H}-27), 0.82$ $(3 \mathrm{H}, \mathrm{t}, J=6.8 \mathrm{~Hz}, \mathrm{H}-29) .{ }^{13} \mathrm{C}-\mathrm{NMR}\left(75 \mathrm{MHz} \mathrm{CDCl}_{3}\right): \delta \mathrm{C}(\mathrm{ppm})$ 71.9 (C-3), 140.9 (C-5), 121.8 (C-6), 140.9 (C-5),36.6 (C-10), 42.4 (C-13),19.9 (C-26),19.5 (C-19),19.1 (C-27),18.9 (C-21),12.1 (C-29), 12.0 (C-18).

Compound 8: 3 $\beta$-Acetoxy-28-hydroxyurs-12-ene, white powder, ${ }^{1} \mathrm{H}-\mathrm{NMR}(500 \mathrm{MHz}, \mathrm{CDCl} 3): \delta \mathrm{H}(\mathrm{ppm}) 5.26(1 \mathrm{H}, \mathrm{t}, \mathrm{J}$ $=3.8 \mathrm{~Hz}, \mathrm{H}-12), 4.09(1 \mathrm{H}, \mathrm{d}, \mathrm{J}=10.3 \mathrm{~Hz}, \mathrm{H}-28 \mathrm{a}), 4.32(1 \mathrm{H}, \mathrm{d}$, $\mathrm{J}=10.8 \mathrm{~Hz}, \mathrm{H}-28 \mathrm{~b}), 2.05$ (3H,s,H-2'), 1.08 (3H,s,H-27), 0.96 (3H,s,H-26),0.93 (3H,s,H-25),0.92 (3H,s,H-29), 0.87 (3H,s,H-30), 0.85 (3H,s,H-23),0.79 (3H,s,H-24). ${ }^{13} \mathrm{C}-\mathrm{NMR}(125 \mathrm{MHz}, \mathrm{CDCl} 3): \delta \mathrm{C}$ 
(ppm) 126.0 (C-12),138.1 (C-13),171.1 (C-1'),28.3 (C-23),23.8 (C-27),21.4 (C-30),21.3 (C-2'),17.3 (C-26), 17.2 (C-29), 16.9 (C-24), 15.7 (C-25), 68.4 (C-28).
Compound 9: Sucrose, light yellow granular crystal, ${ }^{1} \mathrm{H}-\mathrm{NMR}$ (300 MHz,CD $\mathrm{OD}_{3}: \delta_{\mathrm{H}}(\mathrm{ppm}) 4.07(2 \mathrm{H}, \mathrm{d}, J=8.0 \mathrm{~Hz}, \mathrm{H}-1), 4.06$ $\left(2 \mathrm{H}, \mathrm{d}, J=8.1 \mathrm{~Hz}, \mathrm{H}-6^{\prime}\right), 3.6(2 \mathrm{H}, \mathrm{d}, J=9.5 \mathrm{~Hz}, 6-\mathrm{H}), 5.15(1 \mathrm{H}, \mathrm{d}, J$<smiles>O=C1c2cc(CO)cc(O)c2C(=O)c2cccc(O)c21</smiles>

Compound 1<smiles>COc1cc(O)c2c(c1)C(=O)c1cc(C)cc(O)c1C2=O</smiles>

Compound 4<smiles>Oc1ccc(C=Cc2cc(O)cc(O)c2)cc1</smiles>

Compound 6<smiles>Cc1cc(O)c2c(c1)C(=O)c1cccc(O)c1C2=O</smiles>

Compound 2<smiles>Cc1cc(O)c2c(c1)C(=O)c1cc(O)cc(O)c1C2=O</smiles>

Compound 3<smiles>O=c1c(O)c(-c2ccc(O)c(O)c2)oc2cc(O)cc(O)c12</smiles>

Compound 5<smiles>CC[C@H](CC[C@@H](C)[C@H]1CCC2C3CC=C4C[C@@H](O)CC[C@]4(C)C3CC[C@@]21C)C(C)C</smiles>

Compound 7<smiles>OC[C@H]1O[C@@H](O[C@@]2(CO)O[C@H](CO)[C@@H](O)[C@H]2O)[C@H](O)[C@@H](O)[C@@H]1O</smiles>

\section{Compound 9}<smiles>CCCCOC(=O)c1ccccc1C(=O)OCCC=[In]</smiles>

Compound 10

Figure 3. Chemical structures of compounds 1-10. (1) aloe emodin; (2) chrysophanol; (3) emodin; (4), physcion; (5) quercetin; (6) resveratrol; (7) $\beta$-sitosterol; (8) 33-Acetoxy-28-hydroxyurs-12-ene; (9) sucrose; (10) dibutyl phthalate. 
$\left.=3.4 \mathrm{~Hz}, \mathrm{H}-1^{\prime}\right), 3.89(1 \mathrm{H}, \mathrm{d}, J=3.6 \mathrm{~Hz}, \mathrm{H}-4), 3.74\left(1 \mathrm{H}, \mathrm{m}, \mathrm{H}-3^{\prime}\right)$, $3.72\left(1 \mathrm{H}, \mathrm{s}, \mathrm{H}-4^{\prime}\right), 3.52\left(1 \mathrm{H}, \mathrm{s}, \mathrm{H}-2^{\prime}\right), 3.36\left(1 \mathrm{H}, \mathrm{m}, \mathrm{H}-5^{\prime}\right) .{ }^{13} \mathrm{C}-\mathrm{NMR}$ $\left(75 \mathrm{MHz}, \mathrm{CD}_{3} \mathrm{OD}\right): \delta_{\mathrm{C}}(\mathrm{ppm}) 99.8\left(\mathrm{C}-1^{\prime}\right), 71.6\left(\mathrm{C}-2^{\prime}\right), 70.4$ $\left(\mathrm{C}-5^{\prime}\right), 69.8\left(\mathrm{C}-3^{\prime}\right), 67.9\left(\mathrm{C}-4^{\prime}\right), 63.1\left(\mathrm{C}-6^{\prime}\right), 64.4(\mathrm{C}-1), 101.8$ (C2),81.7 (C-5),76.5 (C-4),76.1 (C-3),62.8 (C-6).

Compound 10: Dibutyl phthalate, light yellow oil, ${ }^{1} \mathrm{H}-\mathrm{NMR}$ (300 MHz, $\left.\mathrm{CD}_{3} \mathrm{OD}\right): \delta_{\mathrm{H}}(\mathrm{ppm}) 7.73(2 \mathrm{H}, \mathrm{m}, \mathrm{H}-3, \mathrm{H}-6), 7.64$ $(2 \mathrm{H}, \mathrm{m}, \mathrm{H}-4, \mathrm{H}-5), 1.00\left(6 \mathrm{H}, \mathrm{t}, J=7.4 \mathrm{~Hz}, \mathrm{H}-11, \mathrm{H}-11^{\prime}\right), 4.31(4 \mathrm{H}, \mathrm{t}, \mathrm{J}$ $\left.=6.6 \mathrm{~Hz}, \mathrm{H}-8, \mathrm{H}-8^{\prime}\right), 1.74\left(4 \mathrm{H}, \mathrm{m}, \mathrm{H}-9 \mathrm{H}-9^{\prime}\right), 1.47(4 \mathrm{H}, \mathrm{m}, \mathrm{H}-10, \mathrm{H}-$ $\left.10^{\prime}\right) .{ }^{13} \mathrm{C}-\mathrm{NMR}\left(75 \mathrm{MHz}, \mathrm{CD}_{3} \mathrm{OD}\right): \delta_{\mathrm{C}}(\mathrm{ppm}) 169.3\left(\mathrm{C}-7, \mathrm{C}-7^{\prime}\right) ; \delta_{\mathrm{C}}$ (ppm) 1.00 (C-11,C-11'), 66.7 (C-8,C-8'), 31.7 (C-9,C-9'), 20.3 (C-10,C-10'), 133.6 (C-1,C-2), 132.4 (C-4,C-5), 129.9 (C-3,C-6).

\subsection{Antitumor and cytotoxic effects of ethyl acetate extract of Rumex japonicus Houtt root}

\section{Extract apoptosis effects in HepG2 cells}

For confirmation of anticancer potential of ethyl acetate extract of $R$. japonicus Houtt root, apoptosis induction in HepG2 cancer cells was evaluated in terms of inhibitory effect and results are presented as $\mathrm{IC}_{50}$ values in Table 2. According to Food and Drug Administration (FDA) of Unites States, $\mathrm{IC}_{50}$ value is indicative as measure of half maximal (50\%) inhibitory concentration of potent chemical substance/compound exerting antitumor effect. The $\mathrm{IC}_{50}$ values showing inhibitory effect on HepG2 cancer cells for all extract types, such as EtOAc, aqueous, 20\%, $40 \%$ and $95 \%$ EtOH extracts were $86.7 \mu \mathrm{M}, 111.3 \mu \mathrm{M}, 85.6 \mu \mathrm{M}$, 108.21 $\mu \mathrm{M}, 414.23 \mu \mathrm{M}$ as shown in in Table 2. EtOAc and 20\% $\mathrm{EtOH}$ extracts exhibited comparable inhibitory effects, whereas gradual rises in $\mathrm{IC}_{50}$ values were observed when ethanolic extracts concentration was varied from 40 to $95 \%$. This implied that extract yield increased with corresponding rises in ethanol as extraction solvent and hence cytotoxicity of cancer cells increased from $85.6 \mu \mathrm{M}$ to $414.23 \mu \mathrm{M}$ when ethancolic extracts concentration was varied from 20 to $95 \%$. These results are in coherence with findings of Machana et al. (2012) who reported anticancer effects of Finet \& Gagnep (Polyalthia evecta) against human HepG2 cell line. Authors reported that 50\% ethanol-water crude leaf extract of P. evecta led to moderate inhibitory effect (46.4\%) in human HepG2 cell line. Inhibitory effect caused by antitumor activity of crude extract led to apoptosis which is the essential and reported end point of all anticancer therapies in terms of pharmaco-dynamics (Cummings et al., 2004). Moreover, the programmed cell death of cancer cells caused by antitumor activity of natural plant byproducts metabolites like $R$. japonicus Houtt root provides a balancing effect between uncontrolled cell proliferation and cell death (Bruin \& Medema, 2008; Mondal et al., 2011).

\section{Extract apoptosis effects in U251 cells}

Similar to HepG2 cell line results, the anticancer activity of human cerebral glioma cells (U251) was determined in terms of cytotoxicity. In vitro cytotoxicity evaluation was carried out by MTT assay and results are tabulated in terms of $\mathrm{IC}_{50}$ values in Table 2. Petroleum ether/EtOAc (100:1-1:1) fraction showed $\mathrm{IC}_{50}$ value of $85.6 \mu \mathrm{M}$. The aqueous extract of $R$. japonicus Houtt root exhibited higher inhibitory effect $\left(\mathrm{IC}_{50}\right.$ value: $\left.165.5 \mu \mathrm{M}\right)$ which implied that aqueous extracts were more potent to inhibit proliferation of U251 cancer cell lines. Among ethanolic extracts, $40 \% \mathrm{EtOH}$ extracts showed the most significant inhibitory effect ( $\mathrm{IC}_{50}$ value: $224.3 \mu \mathrm{M}$ ) as compared to those observed in case of $20 \%$ ( $\mathrm{IC}_{50}$ value: $89.1 \mu \mathrm{M}$ ) and $95 \% \mathrm{EtOH}$ extracts $\left(\mathrm{IC}_{50}\right.$ value: $80.1 \mu \mathrm{M})$. Extracts extracted using $>40 \%$ ethanol exhibited corresponding decreasing tendency in inhibitory effect whereas $40 \% \mathrm{EtOH}$ extracts were the most potent to exhibit cytotoxic effects to minimize cancerous cells proliferation. These results are in line with the findings of Alwarsamy \& Ravichandran (2011) who reported anticancer activity of red seaweed (Kappaphycus alvarezii) and the active compounds Fucoidan demonstrated promising results in terms of its oncological significance. The methanolic extract exhibited significantly potent antitumor activity and cytotoxicity in glial cell carcinoma (U251) with $55 \mu \mathrm{g} / \mathrm{mL}$ as the most effective $\mathrm{IC}_{50}$ value.

\subsection{Extract antiproliferative effects in $S K-O V-3 / D D P$ cell line}

For investigating antitumor properties of $R$. japonicus Houtt root extract, cytotoxicity to SKOV-3 cell line was assessed through MTT assay. It was found that cells treated with R. japonicus Houtt root extracts exhibited striking reduction in cell viability. The $\mathrm{IC}_{50}$ values of EtOAc, water, $20 \% \mathrm{EtOH}, 40 \% \mathrm{EtOH}$ and $95 \% \mathrm{EtOH}$ were $65.4 \mu \mathrm{M}, 100.8 \mu \mathrm{M}, 101.4 \mu \mathrm{M}, 288.1 \mu \mathrm{M}$, and $311.36 \mu \mathrm{M}$, respectively. $95 \% \mathrm{EtOH}$ showed the highest cytotoxic effect in SKOV-3 cell line followed by $40 \% \mathrm{EtOH}$ and $20 \% \mathrm{EtOH}$ extracts.

Concentration-dependent reductions in cell viability was found for EtOH extracts. Similar results have been reported by Paramee et al. (2018) for anti-proliferative effects of Kaempferia parviflora extract on SKOV3 ovarian cancer cells. SKOV3 has been reported as high-grade cell line for ovarian cancer and is resistant to many cytotoxic agents. Moreover, majority of the synthetically developed anti-tumor compounds have serious side effects (Cristea et al., 2010), therefore, natural anti-tumor chemical compounds of plant-origin like R. japonicus Houtt root extract might be good candidature for natural cancer therapies

Table 2. $\mathrm{IC}_{50}$ value of the inhibitory effect of extract from roots of Rumex japonicus Houtt on three kinds of cancer cells ( $\mu \mathrm{M}$ ).

\begin{tabular}{lcrr}
\hline \multicolumn{1}{c}{ Sample } & HepG2 & U251 & SKOV-3 \\
\hline Petroleum ether/EtOAc (100:1-1:1) & 86.7 & 85.6 & 65.4 \\
$\mathrm{H}_{2} \mathrm{O}$ & 111.3 & 165.5 & 100.8 \\
$20 \% \mathrm{EtOH}$ & 85.6 & 89.1 & 101.4 \\
$40 \% \mathrm{EtOH}$ & 108.2 & 224.3 & 288.1 \\
$95 \% \mathrm{EtOH}$ & 414.22 & 80.0 & 311.36 \\
\hline
\end{tabular}


exhibiting potent cytotoxic effects to suppress growth of the most commonly diagnosed gynecologic cancer like ovarian cancer as adenocarcinoma.

\subsection{Antitumor activity of R. japonicus Houtt root compounds 1-10}

After evaluation of inhibitory effects of $R$. japonicus Houtt root extract, individual compounds (1-10) were also tested for their antitumor potential on the inhibition of three kinds of cancer cells and results are expressed in terms of $\mathrm{IC}_{50}$ value for each compound shown in Table 3 . The lowest antitumor activity for HepG2 cells was shown by compound 5: quercetin (52.1), compound 4: physcion $(62.3 \mu \mathrm{M})$, compound 6: resveratrol $(68.2 \mu \mathrm{M})$ and compound 8: $3 \beta$-acetoxy-28-hydroxyurs-12-ene $(86.4 \mu \mathrm{M})$. Four compounds: compound 2 (chrysophanol), 7 ( $\beta$-sitosterol), 9 (sucrose), and 10 (dibutyl phthalate) exhibited highly significant and potent antitumor activity $(>100 \mu \mathrm{M})$ for HepG2 cell lines whereas, compounds 3 (aloe emodin), 1 (emodin), and 8 (3 $\beta$-acetoxy-28-hydroxyurs-12-ene) demonstrated antitumor activities of $93.4 \mu \mathrm{M}, 90.8 \mu \mathrm{M}$ and $86.4 \mu \mathrm{M}$, respectively. The possible mechanism for apoptotic effects of anticancer compounds might be ascribed to their modification ability of mitochondrial transmembrane potential and transcriptional activity by means of genetic or nuclear factors and owing to probable inhibition or activation of anti- or proapoptotic proteins (Méndez-Cuesta et al., 2019).

Similarly, cerebral glioma cells (U251) have also exhibited cytotoxicity conferred by ten isolated compounds from $R$. japonicus Houtt root extract. Highest anti-tumor activity of $>100 \mu \mathrm{M}$ was measured for compound 7 ( $\beta$-sitosterol), compound 8 ( $3 \beta$-acetoxy28-hydroxyurs-12-ene), compound 9 (sucrose) and compound 10 (dibutyl phthalate). While, compound 6 (resveratrol) was found with least inhibitory effect $(17.8 \mu \mathrm{M})$ followed compound 3: aloe emodin $(19.8 \mu \mathrm{M})$, compound 4: physcion $(23.5 \mu \mathrm{M})$ and compound 5: quercetin $(23.8 \mu \mathrm{M})$. Conversely, compound 1 (emodin) and compound 2 (chrysophanol) showed moderate cytotoxic effects of $48.3 \mu \mathrm{M}$ and $50.1 \mu \mathrm{M}$ in U251 cancer cell line, respectively.

Individual compounds from 1 to 10 were also assessed for their cytotoxic and tumor suppression effects on ovarian cancer SK-OV-3/DDP cell line and the antitumor potential on inhibition is expressed in terms of $\mathrm{IC}_{50}$ value for each compound shown in Table 3. Similar to cytotoxicity of U251 cell line, compound 7 to 10 , such as $\beta$-sitosterol, $3 \beta$-acetoxy-28-hydroxyurs-12-ene, sucrose and dibutyl phthalate exhibited highly significant tumor suppression activity $(>100 \mu \mathrm{M})$ in SK-OV-3/DDP cell line which implied that extracted and isolated $R$. japonicus Houtt root compounds were effective to inhibit tumor growth of highly resistant cancer cell lines like SK-OV-3/DDP. However, the compound 3 (aloe emodin) showed the least IC $_{50}$ value $(31.3 \mu \mathrm{M})$, while IC50 values of compounds 4 (physcion), 5 (quercetin) and 6 (resveratrol) were $61.1 \mu \mathrm{M}, 47.4 \mu \mathrm{M}$ and $42.1 \mu \mathrm{M}$, respectively which implied that these compounds exhibited moderate cytotoxic activity. The $\mathrm{IC}_{50}$ values of compound 1 (emodin) and compound 2 (chrysophanol) were $62.8 \mu \mathrm{M}$ and $64.2 \mu \mathrm{M}$, respectively. Similar to antitumor compounds of $R$. japonicus Houtt root extract reported in this study, the published literature
Table 3. IC50 value of compound 1-10 on the inhibition of three kinds of cancer cells $(\mu \mathrm{M})$.

\begin{tabular}{cccc}
\hline Compound & HepG2 & U251 & SKOV-3 \\
\hline 1 & $90.8 \pm 4.8$ & $48.3 \pm 1.7$ & $62.8 \pm 3.4$ \\
2 & $>100$ & $50.1 \pm 2.2$ & $64.2 \pm 2.8$ \\
3 & $93.3 \pm 3.2$ & $19.8 \pm 1.6$ & $31.3 \pm 1.2$ \\
4 & $62.3 \pm 2.9$ & $23.5 \pm 0.9$ & $61.0 \pm 2.4$ \\
5 & $52.1 \pm 2.4$ & $23.8 \pm 1.1$ & $47.4 \pm 2.1$ \\
6 & $68.2 \pm 3.2$ & $17.8 \pm 0.8$ & $42.0 \pm 2.4$ \\
7 & $>100$ & $85.3 \pm 3.6$ & $>100$ \\
8 & $86.4 \pm 3.8$ & $>100$ & $>100$ \\
9 & $>100$ & $>100$ & $>100$ \\
10 & $>100$ & $>100$ & $>100$ \\
\hline
\end{tabular}

also reports several anticancer compounds of plant origin like cucurbitacins which are primarily a type of tetracyclic triterpenoids and are found in plants of cucurbitaceae family. The main notable plants as source of cucurbitacins include Trichosanthes cucumerina L. and T. kirilowii Maximowicz (Thai medicinal plants), plant by-products (leaves and fruits) of the Tunisian plant Ecballium elaterium, Hemsleya amabilis roots (ancient Chinese medicinal plant), Cucumis melo L., Balsam pear (Momordica balsamina L.), winter squash (Cucurbita andreana), and bitter cucumber (Citrullus colocynthis) (Florento et al., 2012; Lichota \& Gwozdzinski, 2018; Zyad et al., 2018).

\section{Conclusion}

In this study, water, $20 \% \mathrm{EtOH}, 40 \% \mathrm{EtOH}$ and $95 \% \mathrm{EtOH}$ were obtained by eluting the ethyl acetate fraction of Rumex japonicus Houtt roots. The results showed that ethyl acetate extract and $20 \% \mathrm{EtOH}$ layer had a strong inhibitory effect on HepG2, U251 and SKOV-3 cells, while 95\% EtOH layer had a strong inhibitory effect on U251 cells. The compounds of $20 \%$ $\mathrm{EtOH}, 40 \% \mathrm{EtOH}$ and $95 \% \mathrm{EtOH}$ were isolated and purified. Ten compounds, emodin (1), chrysophanol (2), aloe emodin (3), physcion (4), quercetin (5), reveratorol (6), $\beta$ - sitosterol (7), $3 \beta$ - acetoxy-28-hydroxyurs-12-ene (8), sucrose (9) and dibutyl phalate (10), were identified through NMR spectral data. In vitro cytotoxic assay of 10 compounds showed that compounds $1,3,4,5,6$ and 8 can inhibit the growth of HepG2 cells. Among them, compound 5 can strongly inhibit the growth, and the $\mathrm{IC}_{50}$ value is $52.1 \mu \mathrm{M}$. Compounds 3 , 4,5 and 6 have a strong inhibitory effect on glioma (U251) cells, $\mathrm{IC}_{50}$ values are 19.8, 23.5, 23.8 and $17.8 \mu \mathrm{M}$ respectively, while compounds 1,2 and 7 have a moderate inhibitory effect on glioma (U251) cells growth with $\mathrm{IC}_{50}$ values of 48.3, 50.1 and $85.3 \mu \mathrm{M}$ respectively. Compounds $1,2,3,4,5$ and 6 have a certain inhibitory effect on ovarian cancer (SKOV3) cells, with $\mathrm{IC}_{50}$ values of $62.8,64.2,31.3,61.0,47.4$ and $42.0 \mu \mathrm{M}$, respectively. Moreover, majority of the synthetically developed anti-tumor compounds have serious side effects, therefore, natural anti-tumor chemical compounds of plantorigin like R. japonicus Houtt root extract might be good candidature for natural cancer therapies exhibiting potent cytotoxic effects to suppress growth of the most commonly diagnosed adenocarcinomas. 


\section{References}

Alwarsamy, M., \& Ravichandran, R. (2011). Antiproliferative activity of Kappaphycus Alvarezii extract on three cancer cell lines (NCIH 460, HCT 116 and U 251). Journal of Life Science, 5, 201-205.

Bruin, E. C., \& Medema, J. P. (2008). Apoptosis and non-apoptotic deaths in cancer development and treatment response. Cancer Treatment Reviews, 34(8), 737-749. http://dx.doi.org/10.1016/j. ctrv.2008.07.001. PMid:18722718.

Cristea, M., Han, E., Salmon, L., \& Morgan, R. J. Jr. (2010). Practical considerations in ovarian cancer chemotherapy. Therapeutic Advances in Medical Oncology, 2(3), 175-187. http://dx.doi. org/10.1177/1758834010361333. PMid:21789133.

Cummings, J., Ward, T. H., Ranson, M., \& Dive, C. (2004). Apoptosis pathway-targeted drugs: from the bench to the clinic. Biochimica et Biophysica Acta, 1705(1), 53-66.

Emerson, D., Jones, M., \& Bell, C. B. E. (2007). TPI 287 crosses the blood brain barrier and contributes to antitumor activity in the U251 glioblastoma intracranial tumor model in nude mice. Cancer Research, 67(9), 1441.

Florento, L., Matias, R., Tuaño, E., Santiago, K., Dela Cruz, F., \& Tuazon, A. (2012). Comparison of cytotoxic activity of anticancer drugs against various human tumor cell lines using in vitro cell-based approach. International Journal of Biomedical Science; IJBS, 8(1), 76-80. PMid:23675259.

Guo, L., Wu, J. Z., Han, T., Cao, T., Rahman, K., \& Qin, L. P. (2008). Chemical composition, antifungal and antitumor properties of ether extracts of Scapania verrucosa Heeg. and its endophytic fungus Chaetomium fusiforme. Molecules, 13(9), 2114-2125. http://dx.doi. org/10.3390/molecules13092114. PMid:18830144.

He, Y. Q., Liu, H. N., Li, X. X., \& Wang, G. Y. (2016). Antitumor activity of physcion 8-o- $\beta$-glucopyranoside against cervical cancer by induction of apoptosis. Tropical Journal of Pharmaceutical Research, 15(6), 1145-1150. http://dx.doi.org/10.4314/tjpr.v15i6.5.

Jenks, S., \& Smigel, K. (1995). Updates: cancer drug approved; new leukemia treatment. Journal of the National Cancer Institute, 87(3), 167-170. http://dx.doi.org/10.1093/jnci/87.3.167. PMid:7707401.

Lee, H.-S., Kim, S.-K., Han, J.-B., Choi, H.-M., Park, J.-H., Kim, E.-C., Choi, M. S., An, H. J., Um, J. Y., Kim, H. M., \& Min, B. I. (2006). Inhibitory effects of Rumex japonicus Houtt. on the development of atopic dermatitis-like skin lesions in NC/Nga mice. British Journal of Dermatology, 155(1), 33-38. http://dx.doi.org/10.1111/j.13652133.2006.07303.x. PMid:16792749.

Lee, K. H. (1999). Novel antitumor agents from higher plants. Medicinal Research Reviews, 19(6), 569-596. http://dx.doi.org/10.1002/(SICI)10981128(199911)19:6<569::AID-MED7>3.0.CO;2-9. PMid:10557371.

Lichota, A., \& Gwozdzinski, K. (2018). Anticancer activity of natural compounds from plant and marine environment. International Journal of Molecular Sciences, 19(11), 3533. http://dx.doi.org/10.3390/ ijms19113533. PMid:30423952.

Machana, S., Weerapreeyakul, N., \& Barusrux, S. (2012). Anticancer effect of the extracts from Polyalthia evecta against human hepatoma cell line (HepG2). Asian Pacific Journal of Tropical Biomedicine, 2(5), 368-374. http://dx.doi.org/10.1016/S2221-1691(12)60058-6. PMid:23569932.

Maltese, F., Erkelens, C., Kooy, F., Choi, Y. H., \& Verpoorte, R. (2009). Identification of natural epimeric flavanone glycosides by NMR spectroscopy. Food Chemistry, 116(2), 575-579. http://dx.doi. org/10.1016/j.foodchem.2009.03.023.
Méndez-Cuesta, C. A., Campos, A. L. E., Salinas Sánchez, D., Pérez González, C., \& Pérez Gutiérrez, S. (2019). Cytotoxic and antitumoral activities of compounds isolated from Cucurbitaceae plants. In S. Perveen \& A. Al-Taweel (Eds.), Pharmacognosy: medicinal plants. London: IntechOpen. http://dx.doi.org/10.5772/ intechopen.82213.

Mondal, A., Maity, T. K., Pal, D., Sannigrahi, S., \& Singh, J. (2011). Isolation and in vivo hepatoprotective activity of Melothria heterophylla (Lour.) Cogn. against chemically induced liver injuries in rats. Asian Pacific Journal of Tropical Medicine, 4(8), 619-623. http://dx.doi.org/10.1016/S1995-7645(11)60159-4. PMid:21914539.

Paramee, S., Sookkhee, S., Sakonwasun, C., Na Takuathung, M., Mungkornasawakul, P., Nimlamool, W., \& Potikanond, S. (2018). Anti-cancer effects of Kaempferia parviflora on ovarian cancer SKOV3 cells. BMC Complementary and Alternative Medicine, 18(1), 178. http://dx.doi.org/10.1186/s12906-018-2241-6. PMid:29891015.

Qin, J. L., Shen, W. Y., Chen, Z. F., Zhao, L. F., Qin, Q. P., Yu, Y. C., \& Liang, H. (2017). Oxoaporphine metal complexes (COII, NIII, ZNII) with high antitumor activity by inducing mitochondria- mediated apoptosis and S-phase arrest in HepG2. Scientific Reports, 7(1), 46056. http:// dx.doi.org/10.1038/srep46056. PMid:28436418.

Qin, Q. P., Wang, S. L., Tan, M. X., Luo, D. M., Wang, Z. F., Wei, Q. M., Wu, X.-Y., Zou, B.-Q., \& Liu, Y.-C. (2019). 3-(1H-benzoimidazol2-yl)-chromen-2-ylideneamine platinum(II) and ruthenium(II) complexes exert their high in vitro antitumor activity by inducing S-phase arrest and disrupting mitochondrial functions in SKOV-3/DDP tumor cells. Polyhedron, 157, 219-224. http://dx.doi. org/10.1016/j.poly.2018.10.012.

Steenkamp, V., \& Gouws, M. C. (2006). Cytotoxicity of six South African medicinal plant extracts used in the treatment of cancer. South African Journal of Botany, 72(4), 630-633. http://dx.doi. org/10.1016/j.sajb.2006.02.004.

Thakurta, D. G., Sheikh, N. A., Fan, L. Q., Kandadi, H., Meagher, T. C., Hall, S. J., Kantoff, P. W., Higano, C. S., Small, E. J., Gardner, T. A., Bailey, K., Vu, T., DeVries, T., Whitmore, J. B., Frohlich, M. W., Trager, J. B., \& Drake, C. G. (2015). Humoral immune response against nontargeted tumor antigens after treatment with sipuleucel-T and its association with improved clinical outcome. Clinical Cancer Research, 21(16), 3619-3630. http://dx.doi.org/10.1158/1078-0432. CCR-14-2334. PMid:25649018.

Xie, Q. C., \& Yang, Y. P. (2014). Anti-proliferative of physcion 8-O$\beta$ - glucopyranoside isolated from Rumex japonicus Houtt. on A549 cell lines via inducing apoptosis and cell cycle arrest. BMC Complementary and Alternative Medicine, 14(1), 377. http://dx.doi. org/10.1186/1472-6882-14-377. PMid:25283233.

Yang, H. R., Lee, H., Kim, J. H., Hong, I. H., Hwang, D. H., Rho, I. R., Kim, G. S., Kim, E., \& Kang, C. (2019). Therapeutic effect of Rumex japonicus Houtt. on DNCB-induced atopic dermatitis-like skin lesions in Balb/c mice and human keratinocyte HaCaT cells. Nutrients, 11(3), 573. http://dx.doi.org/10.3390/nu11030573. PMid:30866501.

Zhou, X., Xuan, L., \& Zhang, S. (2005). Study on the chemical constituents from Rumex japonicus Houtt. Zhong Yao Cai, 28(2), 104-105. PMid:15981880.

Zyad, A., Leouifoudi, I., Tilaoui, M., Mouse, H. A., Khouchani, M., \& Jaafari, A. (2018). Natural products as cytotoxic agents in chemotherapy against cancer. In T. A. Celik (Ed.), Cytotoxicity. London: IntechOpen. http://dx.doi.org/10.5772/intechopen.72744. 